\title{
Nanoparticle-Sensitized Photodegradation of Bilirubin and Potential Therapeutic Application
}

Soumik Sarkar, ${ }^{\dagger}$ Abhinandan Makhal, ${ }^{\dagger}$ Sunandan Baruah, ${ }^{\ddagger}$ Mohammad A. Mahmood, ${ }^{\ddagger}$ Joydeep Dutta, ${ }^{\ddagger}, \S$ and Samir K. Pal ${ }^{*}$,

\author{
${ }^{\dagger}$ Department of Chemical, Biological and Macromolecular Sciences, S. N. Bose National Centre for Basic Sciences, Block JD, Sector \\ III, Salt Lake, Kolkata 700 098, India \\ ${ }^{\ddagger}$ Centre of Excellence in Nanotechnology, School of Engineering and Technology, Asian Institute of Technology, Klong Luang, \\ Pathumthani 12120, Thailand \\ ${ }^{\S}$ Chair in Nanotechnology, Water Research Center, Sultan Qaboos University, P.O. Box 17, 123 Al-Khoudh, Sultanate of Oman
}

Supporting Information

ABSTRACT: Bilirubin (BR) is a protective antioxidant; however, when its conjugation and excretion are impaired, as in neonatal and hereditary jaundice, BR accumulates and may cause severe neurotoxicity. Here, we report a novel mechanism for $\mathrm{ZnO}$ nanoparticle (NP)-sensitized BR degradation via defect-mediated nonradiative energy transfer pathway. Among different sizes and shapes, $\mathrm{ZnO}$ particles with diameter of $5 \mathrm{~nm}$ having very high

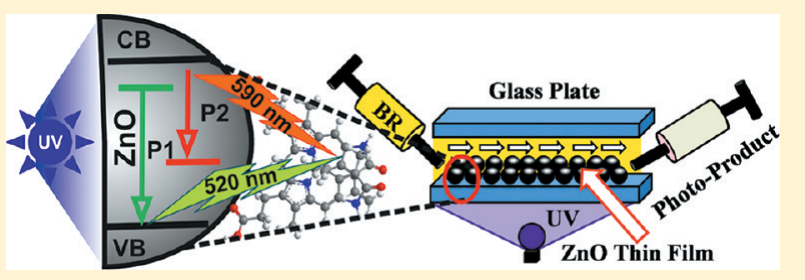
concentration of defect states are found to be the most effective catalyst, which particularly follows a pseudofirst-order kinetics validating the Langmuir-Hinshelwood model of surface catalysis. The nontoxic wide band gap ZnO NPs essentially transmit defect-mediated visible optical radiation, which is not supposed to interfere with the conventional phototherapy process. Therefore, the recyclable $\mathrm{ZnO}$ nanocatalysts essentially invite an added advantage in potential therapeutic applications and/or in a flow-device that has been explored in the present study.

\section{INTRODUCTION}

Bilirubin (BR), the yellow-orange breakdown product of normal heme catabolism in mammalian systems, introduces great biological and diagnostic values. ${ }^{1}$ Both antioxidant and toxic properties have been attributed to $\mathrm{BR}^{2}$, which is normally conjugated with glucuronic acid and then excreted in the bile. However, when its conjugation with glucuronic acid is inhibited, as in neonatal jaundice and in hereditary forms of congenital jaundice, excess BR bind and deposit to various tissues, giving rise to severe hyperbilirubinemia and neurotoxicity. The potential to combat jaundice continues to expand with scientific and technological advancements of the diagnostic tools and treatment methodology. Phototherapy, the most effective treatment for jaundice to date, decreases the BR levels in the blood by changing the ZZ-BR isomer into water-soluble ZE-BR ${ }^{3-5}$ The quantum yield of the photoconversion of $Z Z$ to EE-isomer was found to be dependent on excitation wavelength, decreasing from excitation at 457.9 to $514.5 \mathrm{~nm}$. However, the formation of structural isomer, Z-Lumirubin, was found to follow reverse dependency on the excitation wavelength. ${ }^{7}$ Nevertheless, if the levels of unconjugated BR are very high, one may need to have an exchange transfusion that involves taking away some of the patient's blood and replacing it with a blood transfusion. The decrease in serum BR associated with phototherapy was initially argued to be due to oxidative destruction of the pigment. However, substantial quantities of unconjugated $\mathrm{BR}$ are found in the bile when phototherapy is administered. This fact and the failure to find much of the expected photo-oxidation products in excreta of infants or experimental animals after phototherapy led to discarding the idea that photodegradation is a major pathway. ${ }^{8}$

Nowadays, use of nanoparticles (NPs) in medical science is a promising direction in nanotechnology. ${ }^{9}$ Their applications in drug delivery, ${ }^{10}$ cancer cell diagnostics, ${ }^{11}$ and therapeutics ${ }^{12}$ have been active fields of research. It has been found that the large surface area of the mesoporous anatase $\mathrm{TiO}_{2}$ films, produced by depositing the oxide in colloidal form onto a solid substrate, allows high levels of hemoglobin adsorption. ${ }^{13}$ Zinc oxide $(\mathrm{ZnO})$, a wide band gap $(3.37 \mathrm{eV})$ semiconductor having a high electron-hole binding energy $(60 \mathrm{meV})$, is one of the most attractive and promising materials due to both fundamental research and industrial importance. Although it is less commonly studied and has inferior stability compared to $\mathrm{TiO}_{2}, \mathrm{ZnO}$ is more efficient for degradation of various organic compounds. ${ }^{14} \mathrm{ZnO}$ is also a "green" material that is biocompatible, biodegradable, and nontoxic for medical applications and environmental science. ${ }^{15}$ The excitation of $\mathrm{ZnO}$ with near UV light energy has been shown to be sufficient to initiate various redox reactions at the surface of the semiconductor. Because of the small size of particles and high

Received: February 9, 2012

Revised: April 7, 2012

Published: April 9, 2012 
recombination rate, only a fraction (5\%) of the produced excitons can be utilized to induce redox processes at the interface. The photocatalytic processes using $\mathrm{ZnO}$ and other semiconductors have demonstrated the need to utilize the excitonic recombination in order to achieve higher photocatalytic efficiencies. The photocatalytic property of $\mathrm{ZnO}$ has been widely employed for various applications including antibacterial effect, self-cleaning and self-sterilizing materials, as well as degrading organic contaminants in the environment. ${ }^{16-18}$ Considering the promising applications of $\mathrm{ZnO}$ nanocatalysis, it can be expected that the nanocrystalline $\mathrm{ZnO}$ that possesses self-sterilizing property can also photocatalytically decompose BR when it is adsorbed at the surface of the NPs.

In the present work, we demonstrate the potential use of $\mathrm{ZnO}$ NPs with a strong optical absorption in the near UV region as phototherapeutic agents. The application of defectmediated $\mathrm{ZnO}$ photoluminescence $(\mathrm{PL})$ for the degradation of the surface adsorbed $\mathrm{BR}$, in order to enhance the overall efficiency of the BR photodegradation process, is the major interest in the present study. By using a picosecond-resolved fluorescence technique, we have explored the relative contributions of different defect states (mainly, oxygen vacancy centers) in the overall degradation process. Although the photoexcited charge transfer is widely used in the remediation of chemical contaminants, to the best of our knowledge, no effort has been made to utilize the visible emission of $\mathrm{ZnO}$ in order to degrade the organic contaminants. To investigate the most effective phototherapeutic $\mathrm{ZnO}$ nanostructure, their physicochemical properties, which include size, shape, morphology and surface chemistry, have also been investigated.

\section{EXPERIMENTAL SECTION}

All chemicals used for synthesis were of analytical grade and were not purified further before use. The details of the synthesis of NPs and characterization techniques are given in the following section.

2.1. Synthesis of Catalysts. ZnO NPs were synthesized in a colloidal solution using ethanol, $\mathrm{C}_{2} \mathrm{H}_{5} \mathrm{OH}$ (J. T. Baker), as the solvent. The coprecipitation technique has been reported in previous publications from the group. ${ }^{19}$ Briefly, $40 \mathrm{~mL}$ of $2 \mathrm{mM}$ zinc acetate dihydrate, $\left(\mathrm{CH}_{3} \mathrm{COO}\right)_{2} \mathrm{Zn}, 2 \mathrm{H}_{2} \mathrm{O}$ (Merck), solution was heated at $70{ }^{\circ} \mathrm{C}$ for $30 \mathrm{~min}$. Twenty milliliters of 4 $\mathrm{mM}$ sodium hydroxide, $\mathrm{NaOH}$ (Merck), solution in ethanol was then added and the admixture was hydrolyzed for 2,5 , and $8 \mathrm{~h}$ at $60^{\circ} \mathrm{C}$ to obtain NPs of average diameters of 5,8 , and 10 $\mathrm{nm}$, respectively. $\mathrm{ZnO}$ agglomerates were prepared by adding 1 vol \% of a dispersion of $\mathrm{ZnO}$ NPs (diameter $\sim 5 \mathrm{~nm}$ ) into 50 mmol of an aqueous solution of zinc nitrate hexahydrate, $\mathrm{Zn}\left(\mathrm{NO}_{3}\right)_{2} \cdot 6 \mathrm{H}_{2} \mathrm{O}$ (Aldrich) and hexamethylene tetramine, $\mathrm{C}_{6} \mathrm{H}_{12} \mathrm{~N}_{4}$ (Carlo Erba). The $\mathrm{pH}$ of the reactant solution was adjusted to 8 , and the solution was maintained at $90{ }^{\circ} \mathrm{C}$ for $5 \mathrm{~h}$. The precipitates were then repeatedly washed with deionized water (DI water) and finally with isopropyl alcohol several times, after which the precipitate was dried at $70{ }^{\circ} \mathrm{C}$ for $12 \mathrm{~h}$ to form the powder. $\mathrm{ZnO}$ nanoflowers were synthesized by adding 1 vol \% of a dispersion of $\mathrm{ZnO}$ NPs (diameter $\sim 5 \mathrm{~nm}$ ) into 25 mmol of an aqueous solution of zinc nitrate hexahydrate and hexamethylene tetramine. The $\mathrm{pH}$ of the reactant solution was adjusted to 12 and maintained at $90{ }^{\circ} \mathrm{C}$ for $5 \mathrm{~h}$. The nanoflowers were then washed with DI water a number of times and finally with isopropyl alcohol through centrifugation. The powder was dried at $70{ }^{\circ} \mathrm{C}$ for $12 \mathrm{~h}$. Polycrystalline $\mathrm{ZnO}$
NP thin films (used in the flow-device) were grown on glass substrates through spray pyrolysis technique. Prior to deposition, the substrates were cleaned through sonication for 15 min successively in soap water, acetone, and DI water and dried at $90{ }^{\circ} \mathrm{C}$. Twenty millimolar zinc acetate solution was sprayed on substrates maintained at $300{ }^{\circ} \mathrm{C}$ with a spray gun for $30 \mathrm{~min}$ wherein air pressure and solution flow rate were adjusted at 1.0 bar and $10 \mu \mathrm{L} / \mathrm{s}$, respectively. The spray gun was placed at a distance of $25-30 \mathrm{~cm}$ from the substrates.

2.2. Characterization. Transmission electron microscopy (TEM) grids were prepared by applying a drop of the $\mathrm{ZnO}$ samples to carbon-coated copper grids. Particle sizes were determined from micrographs recorded at a magnification of $100000 \times$ using an FEI (Technai S-Twin, operating at $200 \mathrm{kV}$ ) instrument. Steady-state absorption and emission spectra were measured with a Shimadzu UV-2450 spectrophotometer and Jobin Yvon Fluoromax-3 fluorimeter, respectively. All the PL transients were measured using the picosecond-resolved timecorrelated single photon counting (TCSPC) technique, via a commercially available picosecond diode laser-pumped (LifeSpec-ps) fluorescence spectrophotometer from Edinburgh Instruments, U.K. Picosecond excitation pulses from the picoquant diode laser were used at $375 \mathrm{~nm}$ with an instrument response function (IRF) of 60 ps. A microchannel-platephotomultiplier tube (MCP-PMT, Hammamatsu) was used to detect the PL from the sample after dispersion through a monochromator. For all transients, the polarizer on the emission side was adjusted to be at $55^{\circ}$ (magic angle) with respect to the polarization axis of the excitation beam. Native and BR-capped UV irradiated human serum albumin (HSA) structures were carried out by circular dichroism (CD) measurements in a JASCO 815 spectro-polarimeter at $20 \pm$ $0.1{ }^{\circ} \mathrm{C}$. The scan speed of the measurements was $100 \mathrm{~nm} / \mathrm{min}$, and each spectrum was the average of five scans. The spectral data were acquired over the range of 330-220 nm using a 2 $\mathrm{mm}$ path length cuvette.

2.3. Data Analysis. Curve fitting of observed fluorescence transients was carried out using a nonlinear least-squares fitting procedure to a function $\left(X(t)=\int{ }_{0}^{t} E\left(t^{\prime}\right) R\left(t-t^{\prime}\right) \mathrm{d} t^{\prime}\right)$ comprised of convolution of the IRF $(E(t))$ with a sum of exponentials $\left(R(t)=A+\sum_{i=1}^{N} B_{i} \mathrm{e}^{-\left(t / \tau_{i}\right)}\right)$ with pre-exponential factors $\left(B_{i}\right)$, characteristic lifetimes $\left(\tau_{i}\right)$, and a background $(A)$. Relative concentration in a multiexponential decay is expressed as $c_{n}=$ $\left(B_{n} /\left(\sum_{i=1}^{N} B_{i}\right) \times 100\right)$ The average lifetime (amplitudeweighted) of a multiexponential decay ${ }^{20}$ is expressed as $\tau_{\text {av }}=$ $\sum_{i=1}^{N} c_{i} \tau_{i}$

2.4. Förster Resonance Energy Transfer (FRET) Calculations. In order to estimate the FRET efficiency of the donor $(\mathrm{ZnO})$ and hence to determine the distance of donor-acceptor pairs, we used the following methodology: ${ }^{20}$ The Förster distance $\left(R_{0}\right)$ is given by

$$
R_{0}=0.211 \times\left[\kappa^{2} n^{-4} Q_{D} J(\lambda)\right]^{1 / 6} \quad(\text { in } \AA)
$$

where, $\kappa^{2}$ is a factor describing the relative orientation in space of the transition dipoles of the donor and acceptor. For donor and acceptors that randomize by rotational diffusion prior to energy transfer, the magnitude of $\kappa^{2}$ is assumed to be $2 / 3$. The refractive index $(n)$ of the medium is measured to be 1.4. $Q_{D}$, the integrated quantum yield of the donor in the absence of acceptor, is measured to be $3.8 \times 10^{-3} . J(\lambda)$, the overlap integral, which expresses the degree of spectral overlap between 
the donor emission intensity (normalized to unit area) ${ }^{21}$ and the acceptor absorption, is given by

$$
J(\lambda)=\frac{\int_{0}^{\infty} F_{D}(\lambda) \varepsilon_{\mathrm{A}}(\lambda) \lambda^{4} \mathrm{~d} \lambda}{\int_{0}^{\infty} F_{\mathrm{D}}(\lambda) \mathrm{d} \lambda}
$$

where $F_{D}(\lambda)$ is the fluorescence intensity of the donor in the wavelength range of $\lambda$ to $\lambda+\mathrm{d} \lambda$ and is dimensionless. $\varepsilon_{\mathrm{A}}(\lambda)$ is the extinction coefficient (in $\mathrm{M}^{-1} \mathrm{~cm}^{-1}$ ) of the acceptor at $\lambda$. If $\lambda$ is in nanometers, then $J(\lambda)$ is in units of $\mathrm{M}^{-1} \mathrm{~cm}^{-1} \mathrm{~nm}^{4}$. The estimated value of the overlap integral is $9.91 \times 10^{14}$.

Once the value of $R_{0}$ is known, the donor-acceptor distance $(r)$ can be easily calculated using the following expression:

$$
r^{6}=\frac{\left[R_{0}^{6}(1-E)\right]}{E}
$$

where $E$ is the efficiency of energy transfer. The transfer efficiency is measured using the relative fluorescence lifetime of the donor, in the absence $\left(\tau_{\mathrm{D}}\right)$ and presence $\left(\tau_{\mathrm{DA}}\right)$ of the acceptor.

$$
E=1-\frac{\tau_{\mathrm{DA}}}{\tau_{\mathrm{D}}}
$$

From the average lifetime calculation for the $\mathrm{ZnO}-\mathrm{BR}$ adduct, we obtain the effective distance between the donor and the acceptor $\left(r_{\mathrm{DA}}\right)$, using eqs 3 and 4 .

2.5. Photocatalytic Study. In the photocatalytic study, a fiber-optic based optical system was used for the measurement of light-induced chemical processes with spectroscopic precision. The efficacy of the designed system was previously established through a detailed study of vitamin B2 (riboflavin) photodegradation in aqueous phase. ${ }^{22}$ In order to achieve controlled and preferential excitation of $\mathrm{ZnO} \mathrm{NPs}$, we have used a $320 \mathrm{~nm}$ high-pass optical filter placed on a homemade UV bath (60 W; normally used for water purification). The light above $320 \mathrm{~nm}$ is so chosen that it will be preferentially absorbed by ZnO NPs and will avoid direct photodegradation of BR by deep UV light. Under selective UV radiation, the degradation course was monitored by recording the absorption peak of BR (at $450 \mathrm{~nm}$ ) at $10 \mathrm{~s}$ intervals, using SPECTRA SUITE software supplied by Ocean Optics, and plotting it against the time of photoirradiation. Note that in the present study, we are interested in exploring the long-time photodegradation of BR (for several minutes).

\section{RESULTS AND DISCUSSION}

The morphological and structural investigation of photocatalysts has been carried out by TEM and scanning electron microscopy (SEM). Representative TEM images of various sized $(5,8$, and $10 \mathrm{~nm}) \mathrm{ZnO}$ NPs (Figure 1a-d) and SEM images of $\mathrm{ZnO}$ agglomerates (200-500 nm, Figure 1e) and $\mathrm{ZnO}$ nanoflowers (300-400 nm, Figure 1f) are shown. Highresolution TEM (HRTEM) image of $5 \mathrm{~nm}$ sized $\mathrm{ZnO}$ particles are shown in Figure 1a, which confirms that the particles are fairly monodispersed and highly crystalline in nature. The lattice fringe width of $0.28 \mathrm{~nm}$ indicates the (100) plane of the wurtzite structure of $\mathrm{ZnO}$ nanocrystals. ${ }^{23}$

The UV-visible absorption spectra of different $\mathrm{ZnO}$ colloidal solutions employed in the present study are depicted in Figure 2a. The ZnO NPs exhibit a sharp absorbance onset at about $355 \mathrm{~nm}(3.49 \mathrm{eV})$, reflecting their uniform size. The peak of the exciton absorption band gradually shifts from 355 to 375
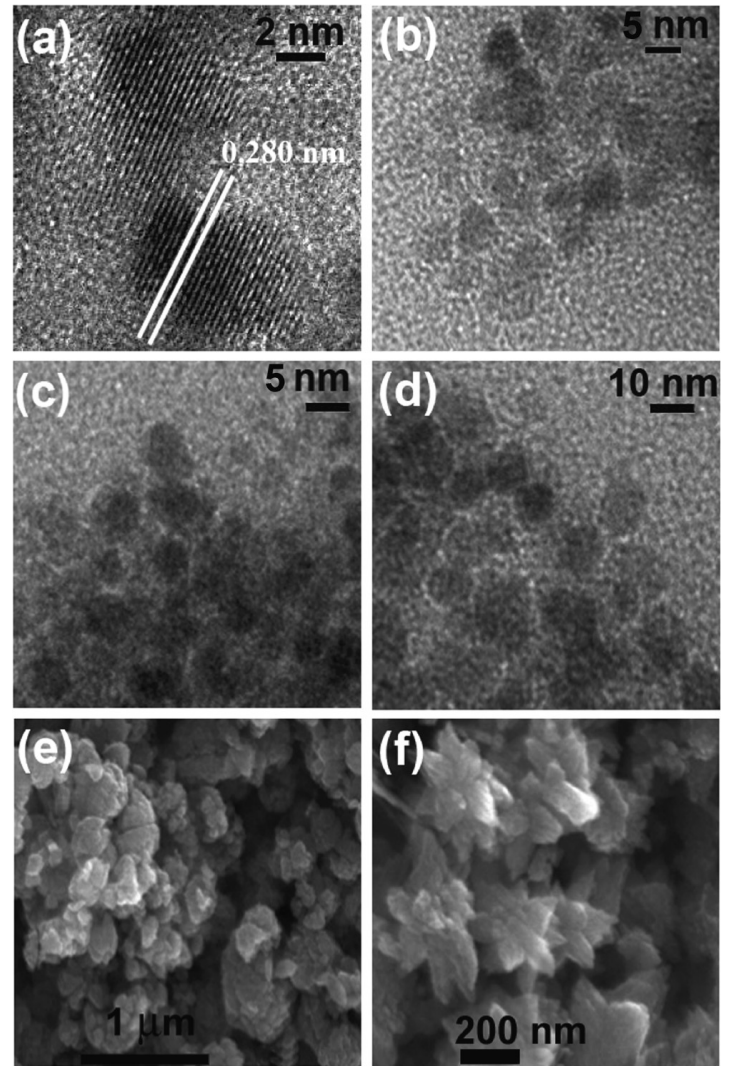

Figure 1. (a) High-magnification TEM image of $\mathrm{ZnO} \mathrm{NP}(5 \mathrm{~nm})$. Low-magnification TEM images of various $\mathrm{ZnO}$ nanocrystallites: (b) $\mathrm{ZnO} \mathrm{NP}(5 \mathrm{~nm}),(\mathrm{c}) \mathrm{ZnO} \mathrm{NP}(8 \mathrm{~nm})$, and (d) $\mathrm{ZnO} \mathrm{NP}(10 \mathrm{~nm})$. SEM images of (e) $\mathrm{ZnO}$ agglomerates and (f) $\mathrm{ZnO}$ nanoflowers.

$\mathrm{nm}(3.49-3.31 \mathrm{eV})$ as the size increases both in diameter and length. The room-temperature PL spectra from the NP samples all contain a narrow UV band centered near $368 \mathrm{~nm}$, which is due to exciton recombination and a broad green band centered at $550 \mathrm{~nm}$ (see Figure 2b). These broad emission bands originate from the surface defect centers, indicating the presence of considerable oxygen vacancies in the crystal lattice of $\mathrm{ZnO} \mathrm{NPs}$, whereas, $\mathrm{ZnO}$ agglomerate and nanoflower structures contain very little of these defects. The broad green emission band is composed of two emission bands (P1 and P2, shown in Figure $2 \mathrm{c}$ as green and red dotted lines, respectively) typically associated with oxygen deficiency. The doubly charged oxygen vacancies $\left(V_{\mathrm{O}}^{+2}\right)$, created by capture of a hole by the singly charged vacancy center $\left(V_{\mathrm{O}}^{+}\right)$in a depletion region, leads to the $\mathrm{P} 2$ emission line. The charged center $\left(V_{\mathrm{O}}^{+}\right)$in the absence of a depletion region turns into a neutral center $\left(V_{\mathrm{O}}{ }^{\times}\right)$ upon the capture of an electron (n-type $\mathrm{ZnO}$ ) from the conduction band, which then recombines with a hole in the valence band giving rise to the P1 emission. ${ }^{24}$

As shown in the Figure 2d, defect-mediated green emission is suppressed upon gradual addition of BR to the system. Previous study has shown that $\mathrm{ZnO} \mathrm{PL}$ is sensitive to the presence of various adsorbates such as $\mathrm{O}_{2}, \mathrm{H}_{2}$, and $\mathrm{CO}$, which also decreases PL intensity; however, methanol and formic acid adsorption increased it. ${ }^{25,26}$ Herein, we propose FRET from a donor $\mathrm{ZnO} \mathrm{NP}$ to $\mathrm{BR}$ acceptor, which is responsible for the observed inhibition of emission bands. FRET in combination with Förster theory has become an invaluable tool for the assessment of distances in numerous biomolecular assem- 

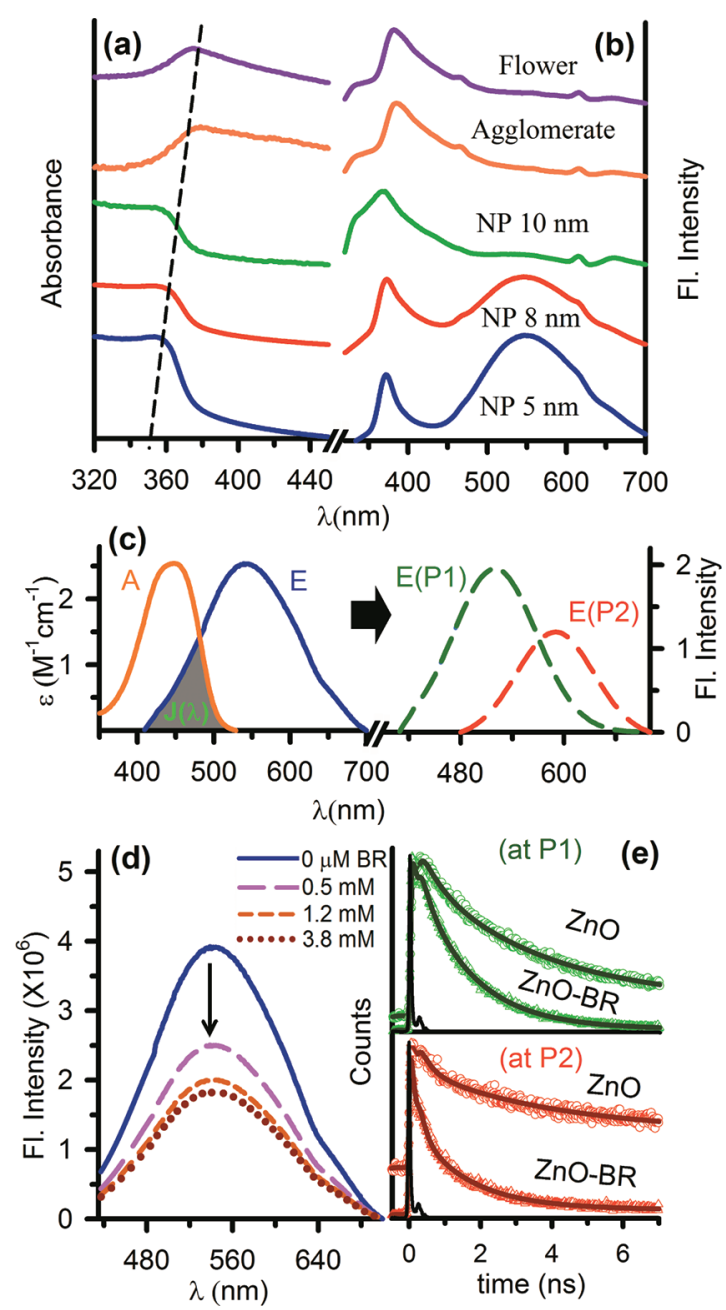

Figure 2. Steady-state (a) absorption spectra and (b) emission spectra (excitation at $320 \mathrm{~nm}$ ) of $\mathrm{ZnO}$ catalysts with various sizes and shapes. (c) Absorption spectra of BR (A, orange) and emission spectra (excitation at $375 \mathrm{~nm}$, Raman extracted) of $\mathrm{ZnO} \mathrm{NP}$ (E, blue) are shown. A typical emission band is composed of two components, P1 and P2 (see text), as shown for the emission curve. An overlapping zone between emission of $\mathrm{ZnO} \mathrm{NP}$ and absorption of acceptor $\mathrm{BR}$ is indicated by the gray shaded zone. (d) PL quenching of $\mathrm{ZnO}$ NPs with the increasing concentration of BR. (e) The picosecond-resolved fluorescence transients of $\mathrm{ZnO} \mathrm{NP}$, in the absence and in the presence of acceptor BR (excitation at $375 \mathrm{~nm}$ ) collected at P1 (green, $520 \mathrm{~nm}$ ) and P2 (red, $590 \mathrm{~nm})$, are shown.

blies. $^{20,27,28}$ The FRET process is based on the concept of treating an excited donor as an oscillating dipole that can undergo energy exchange with a second dipole having a similar resonance frequency. ${ }^{20}$ In principle, if the fluorescence emission spectrum of the donor molecule overlaps the absorption spectrum of an acceptor molecule, and the two are within a minimal distance from one another $(1-10 \mathrm{~nm})$, the donor can directly transfer its excitation energy to the acceptor via exchange of a virtual photon. The consideration of Dexter mechanism (orbital overlap) via an exchange process associated with the transfer of single charge carriers can be excluded in this study as it is a short-range mechanism $(1-10 \AA) .{ }^{29}$ The spectral overlap of the defect-mediated PL band with that of the BR absorption is shown in Figure 2c. It has been reported that the emission center around $590 \mathrm{~nm}$ comes from defect states near the surface layer, while the shorter wavelength at $520 \mathrm{~nm}$ comes from defects near the bulk of the NPs. Picosecond-resolved fluorescence study (excitation at $375 \mathrm{~nm}$ ) was performed by monitoring decays at the P1 and P2 peaks. The faster excited state lifetime of the $\mathrm{ZnO}-\mathrm{BR}$ adduct with respect to that of the free $\mathrm{ZnO}$ NP is clearly noticeable from Figure $2 \mathrm{e}$. The baseline upliftment comes from the long lifetime component, which could not be registered within our experimental time window. The details of the spectroscopic parameters and the fitting parameters of the fluorescence decays are tabulated in Table 1.

Table 1. Picosecond-Resolved Luminescence Transients of $\mathrm{ZnO}$ NPs in the Absence and in the Presence of $\mathrm{BR}^{a}$

$\begin{array}{ccccc}\text { samples } & \tau_{1}(\mathrm{~ns}) & \tau_{2}(\mathrm{~ns}) & \tau_{3}(\mathrm{~ns}) & \begin{array}{c}\tau_{\text {avg }} \\ (\mathrm{ns})\end{array} \\ \mathrm{ZnO} \mathrm{NP}(520 \mathrm{~nm}) & 32.3 & 3.29 & 0.497(24 \%) & 5.73 \\ & (10.7 \%) & (65.3 \%) & & \\ \mathrm{ZnO} \mathrm{NP+BR} & 15.8(1.1 \%) & 1.48 & 0.216 & 1.24 \\ (520 \mathrm{~nm}) & & (67.7 \%) & (31.2 \%) & \\ \mathrm{ZnO} \mathrm{NP}(590 \mathrm{~nm}) & 38.9 & 3.19 & 0.271(40 \%) & 12.23 \\ & (28.5 \%) & (31.5 \%) & & \\ \mathrm{ZnO} \mathrm{NP}+\mathrm{BR} & 24.3(3 \%) & 1.45 & 0.153 & 1.29 \\ (590 \mathrm{~nm}) & & (32.2 \%) & (64.8 \%) & \end{array}$

${ }^{a_{T}}$ The emissions from $\mathrm{ZnO}$ NPs (probing at 520 and $590 \mathrm{~nm}$ ) were detected with a $375 \mathrm{~nm}$ laser excitation. Numbers in the parentheses indicate relative weightage.

From FRET calculations (see Experimental Section), the effective distances between the donor and the acceptor $\left(r_{\mathrm{DA}}\right)$ are determined to be 1.48 and $1.28 \mathrm{~nm}$ for P1 and P2 states, respectively, which supports the general understanding that $V_{\mathrm{O}}^{+2}$ centers are closer to the surface attached BR compared to $V_{\mathrm{O}}{ }^{+}$centers. It is to be noted that the smaller value of $r_{\mathrm{DA}}$ compared to the radius of the $\mathrm{ZnO} \mathrm{NPs}(\sim 2.5 \mathrm{~nm}$; Figure 1a) can be rationalized from the fact that the origin of the PL peaking at $\mathrm{P} 1$ and $\mathrm{P} 2$ arise essentially from surface defects in the $\mathrm{ZnO}$ NPs. ${ }^{30}$ The energy transfer efficiencies of the two states are calculated to be $78 \%$ and $89 \%$, respectively. Since $V_{\mathrm{O}}^{+2} / V_{\mathrm{O}}{ }^{+}$states are located near the surface, greater energy transfer efficiency via doubly charged vacancy centers is well justified. Following the demonstration of relative contributions from different defect states in the energy transfer process, now we proceed to study the effect of transferred energy between the FRET pairs, which finally led to efficient BR degradation in the area of nanocatalysis.

The model photocatalytic reaction investigated in this study is the decomposition of BR (purchased from Sigma-Aldrich) adsorbed at the surface of $\mathrm{ZnO}$ NPs. BR has optical absorption in the UV-visible regions (Figure 2c) and displays a significant decomposition rate in the presence of colloidal $\mathrm{ZnO}$ solutions. There are some previous studies from our group where photolysis of a test contaminant has been probed in a colloidal semiconductor system. ${ }^{31,32}$ BR adsorption on nanostructured hydroxyapatite coating was previously investigated by Yang et al. using quartz crystal microbalance, and the photocatalytic degradation of adsorbed BR under UV irradiation was studied. ${ }^{33}$ It is obvious that the higher the energy transfer from the surface of the $\mathrm{ZnO}$ semiconductor, the faster the degradation of the surface-attached BR. The results of BR degradation in the presence and the absence of $\mathrm{ZnO}$ catalysts under UV light are shown in Figure 3a, where the relative concentration $\left(C_{t} / C_{0}\right)$ of $\mathrm{BR}$ in solution is plotted against $\mathrm{UV}$ irradiation time. Blank experiments performed for several hours in the absence of catalysts and/or without UV irradiation have 

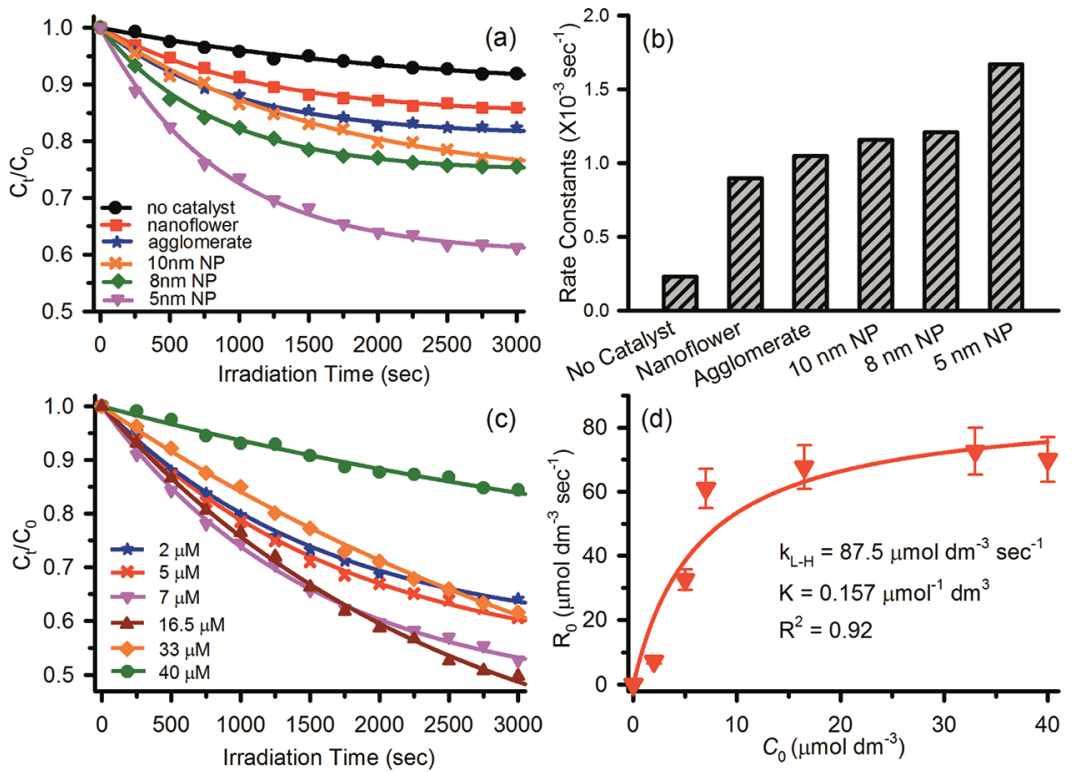

Figure 3. (a) Plot of relative concentration $\left(C_{t} / C_{0}\right)$ versus UV irradiation time for the degradation of BR (monitored at $450 \mathrm{~nm}$ ), in the presence of various $\mathrm{ZnO}$ nanocrystallites. (b) Respective rate constants showing $5 \mathrm{~nm}$ particles are the most effective catalysts. $(\mathrm{c})\left(C_{t} / C_{0}\right)$ versus $t$ with various BR concentrations and (d) Langmuir-Hinshelwood (L-H) plot (with 10\% error bar), are shown. Langmuir-Hinshelwood (L-H) rate constant, Langmuir adsorption coefficient $(K)$ and regression coefficients $\left(R^{2}\right)$ are also provided.

only resulted in a negligible change in the BR concentration. In this respect, BR degradation in the presence of UV leads to photoproduct revealing characteristic absorbance and emission peaks of methylvinylmaleimide (MVM), which is very much in agreement with a previous report (see Supporting Information, Figure S1). ${ }^{34}$ However, the other photoproducts such as configurational (principally, ZE-BR) and structural isomer (Lumirubin) are also expected since $\mathrm{ZnO}$ defect-mediated optical radiation transfer is not supposed to interfere with the conventional phototherapy process.

In order to attain the most efficient photodegradation, the process has been investigated for a range of different $\mathrm{ZnO}$ morphologies and sizes, among which $\mathrm{ZnO}$ particles with diameter of $5 \mathrm{~nm}$ are found to be the most efficient catalysts, as shown in the histogram (Figure $3 b$ ). Particle shape and size are important parameters for catalysis in general since they directly affect the specific surface influence of a catalyst. ${ }^{35-37}$ With a smaller particle size, the surface-to-volume ratio and the number of active surface sites increases, which concomitantly increases the surface charge carrier transfer rate during photocatalysis. $^{38,39}$ In order to increase the quantum yield of nanocrystalline photocatalysts, the $\mathrm{e}^{-} / \mathrm{h}^{+}$recombination has to be reduced. An effective method to achieve the separation of $\mathrm{e}^{-}$ and $\mathrm{h}^{+}$is to introduce defects into the $\mathrm{ZnO}$ lattice. Different mechanisms and different effects of native defects have been proposed in the literature for efficient photocatalytic dye degradation by the use of $\mathrm{ZnO}^{40-42}$ It is obvious that the degradation rate increases with increasing $\mathrm{ZnO}$ concentration, which allows greater contact between organic molecules and photocatalytic active sites (see Supporting Information Figure S2). Now, by keeping the surface concentration fixed, BRdecomposition reaction rate $\left(R_{0}\right)$ is observed to be a function of the substrate initial concentration $\left(C_{0}\right)$. All the photodegradation curves were found to follow a first-order exponential equation, and the results of photocatalytic degradation of $\mathrm{BR}$ over nanocrystalline $\mathrm{ZnO}$ of varying particle sizes and shapes are presented in Table 2. The rate of
Table 2. The Kinetics Parameters ${ }^{a}$ for the Photodegradation of $\mathrm{BR}$ in the Absence and Presence of $\mathrm{ZnO}$

\begin{tabular}{|c|c|c|c|}
\hline samples & $k\left(\mathrm{~s}^{-1}\right)$ & $\begin{array}{l}\text { total amount of degradation (A \%) } \\
\text { (in } 3000 \mathrm{~s})\end{array}$ & $R^{2}$ \\
\hline \multicolumn{4}{|c|}{ ZnO Size and Morphology Dependent Study (10 $\mu \mathrm{M}$ BR $)$} \\
\hline no catalyst & $2.3 \times 10^{-4}$ & 8 & 0.82 \\
\hline $\begin{array}{l}\mathrm{ZnO} \\
\text { nanoflower }\end{array}$ & $8.98 \times 10^{-4}$ & 18 & 0.78 \\
\hline $\begin{array}{l}\mathrm{ZnO} \\
\text { agglomerate }\end{array}$ & $1.05 \times 10^{-3}$ & 14 & 0.88 \\
\hline $\begin{array}{l}\mathrm{ZnO} \mathrm{NP} \\
(\sim 10 \mathrm{~nm})\end{array}$ & $1.16 \times 10^{-3}$ & 23 & 0.85 \\
\hline $\begin{array}{l}\mathrm{ZnO} \mathrm{NP} \\
\quad(\sim 8 \mathrm{~nm})\end{array}$ & $1.21 \times 10^{-3}$ & 25 & 0.89 \\
\hline $\begin{array}{l}\mathrm{ZnO} \mathrm{NP} \\
\quad(\sim 5 \mathrm{~nm})\end{array}$ & $1.67 \times 10^{-3}$ & 37 & 0.86 \\
\hline \multicolumn{4}{|c|}{ BR Concentration Dependent Study (50 $\mu \mathrm{M} \mathrm{ZnO})$} \\
\hline $2 \mu \mathrm{MBR}$ & $3.6 \times 10^{-4}$ & 36 & 0.92 \\
\hline $5 \mu \mathrm{MBR}$ & $6.5 \times 10^{-4}$ & 39 & 0.94 \\
\hline $7 \mu \mathrm{M} \mathrm{BR}$ & $8.7 \times 10^{-4}$ & 47 & 0.93 \\
\hline $16.5 \mu \mathrm{M}$ BR & $4.1 \times 10^{-4}$ & 50 & 0.97 \\
\hline $33 \mu \mathrm{M}$ BR & $2.2 \times 10^{-4}$ & 39 & 0.98 \\
\hline $40 \mu \mathrm{M}$ BR & $1.7 \times 10^{-4}$ & 17 & 0.99 \\
\hline
\end{tabular}

${ }^{a}$ Kinetic constants $(k)$, the percentages of total photodegradation $(A)$ and regression coefficient $\left(R^{2}\right)$. A $320 \mathrm{~nm}$ high-pass optical filter was used in the study.

photocatalytic decomposition of the BR molecules is typically fitted with the Langmuir-Hinshelwood (L-H) kinetics model: ${ }^{43}$

$$
R_{0}=\frac{\mathrm{d} C_{0}}{\mathrm{~d} t}=\frac{k_{\mathrm{L}-\mathrm{H}} K C_{0}}{1+K C_{0}}
$$

where $C_{0}$ is the initial concentration of the BR solution, $t$ is the $\mathrm{UV}$ irradiation time, $k_{\mathrm{L}-\mathrm{H}}$ is the Langmuir-Hinshelwood rate constant, and $K$ is the Langmuir adsorption coefficient of the $\mathrm{BR}$ molecules. If the initial concentration of the BR solution is 


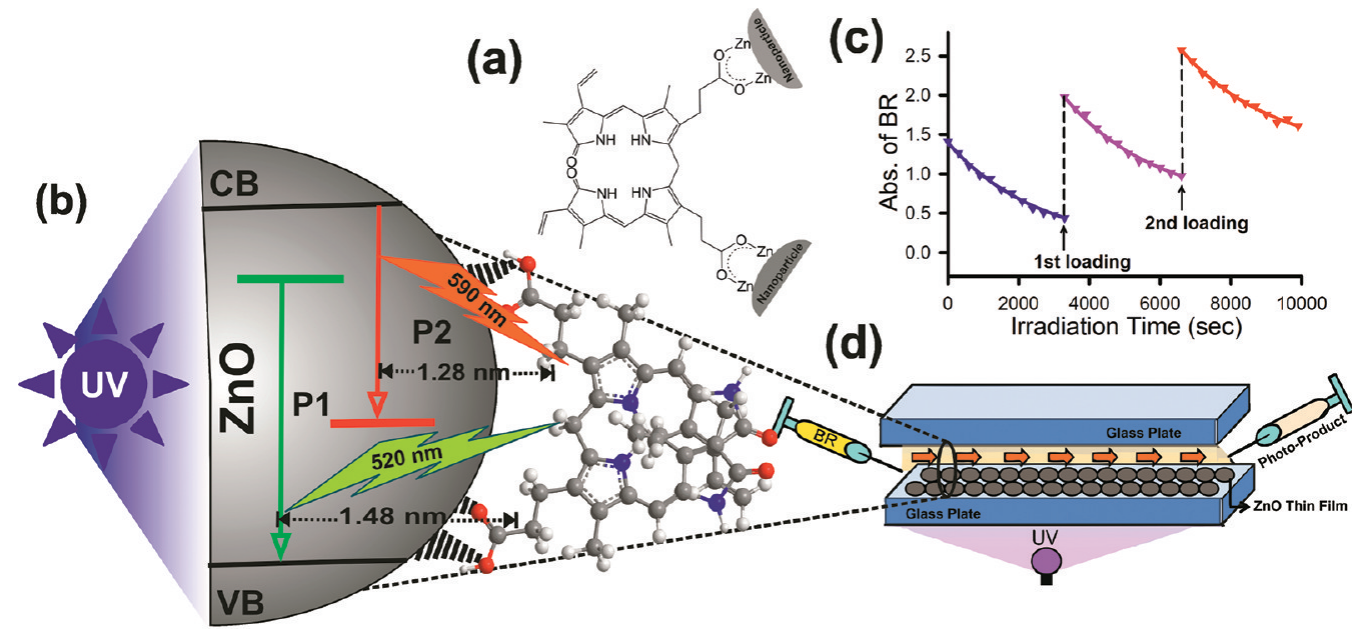

Figure 4. (a) Molecular structure of $\mathrm{BR}$ and its attachment to the surface of $\mathrm{ZnO}$ NPs. (b) Representation of the ZnO NP-BR adducts depicting FRET dynamics from different oxygen vacancy centers of ZnO NPs (gray big ball) to surface-adsorbed BR (shown in ball-stick model) molecules. (c) Photocatalytic degradation curves (abs. vs $t$ ) of $\mathrm{BR}$ in $\mathrm{ZnO}$ suspension under UV-light irradiation for three repeated cycles. (d) A schematic representation of our designed flow-device using $\mathrm{ZnO} \mathrm{NPs}$ as phototherapeutic agents.

sufficiently low $\left(K C_{0} \ll 1\right)$, eq 5 can be simplified to an apparent first-order equation:

$$
R_{0}=k_{\mathrm{L}-\mathrm{H}} K C_{0}=k_{\text {app }} C_{0}
$$

where $k_{\text {app }}$ is the apparent first-order rate constant. If the initial concentration of the BR solution is sufficiently high $\left(K C_{0} \gg 1\right)$, eq 5 can be simplified to a zero-order rate equation:

$$
R_{0}=k_{\mathrm{L}-\mathrm{H}}
$$

In this experiment, $\mathrm{BR}$ degradation on the $\mathrm{ZnO} \mathrm{NP}$ surface was found to exactly follow the above phenomenon (Figure $3 \mathrm{c}, \mathrm{d}$ ). By fitting the observed $R_{0}$ versus $C_{0}$ curve (using eq 5 as the fitting equation) for the degradation of $\mathrm{BR}$ in the presence of the same concentration of $\mathrm{ZnO}$, the obtained values of $k_{\mathrm{L}-\mathrm{H}}$ and $K$ are $87.5 \mu \mathrm{mol} \mathrm{dm}^{-3} \mathrm{~s}^{-1}$ and $0.157 \mu \mathrm{mol}^{-1} \mathrm{dm}^{3}$, respectively. The pattern of the curves and the values of the fitting parameters fall in the range of the observed literature data for various photocatalytic studies. ${ }^{44}$

The capability of being reusable is one of the most pivotal criteria for an ideal photocatalyst. ${ }^{45,46}$ With the successful exhibition of these NPs as effective photocatalysts, the regeneration of the NPs in the process of photocatalysis was further confirmed from the similar rate of photodegradation of $\mathrm{BR}$ for three cycles. The results, given in Figure $4 \mathrm{c}$, demonstrate that these NPs can indeed serve as highly effective and convenient recyclable photocatalysts. The change in concentration of BR solution for multiple steps also fit very well to the exponential decay profile with regression coefficient, $R^{2} \geq 0.94$, and the rate constants were determined to be almost the same $\left(\sim 3.8 \times 10^{-4} \mathrm{~s}^{-1}\right)$ for the multiple cycles.

In order to apply our findings into a model application, we have designed a flow-device consisting of two glass plates (separated by a spacer) as shown in Figure $4 \mathrm{~d}$. Polycrystalline $\mathrm{ZnO} \mathrm{NP}$ (diameter $\sim 5 \mathrm{~nm}$ ) thin films were grown on one of the glass plates by spray pyrolysis technique, and BR solution was passed through the channel at a very slow flow rate $(2 \mathrm{~mL} /$ $30 \mathrm{~min}$ ). The whole system was kept over a UV source, and the total amount of BR degradation was found to be $4 \%$ and $37 \%$ in the absence and presence of $\mathrm{ZnO}$ NPs, respectively, under the same flow rate condition. Therefore, it is clear from our results that $\mathrm{BR}$ can be degraded by careful utilization of nonradiative recombination in $\mathrm{ZnO}$. We like to emphasize two potential advantages of using such an NP-based flow-device in the purification of BR-containing blood. First, the recyclable NPs need minimum recharging that can easily be done by passing an aqueous solution with $\mathrm{pH}$ at 8 followed by deionized water. Second, the exposure of the BR-containing blood to the harmful UV radiation can be avoided using the high pass filter action of the NPs layer. Although $\mathrm{Zn}^{2+}$ is a biologically trace element, cytotoxic response of a critical $\mathrm{ZnO}$ concentration $(3.75 \mathrm{ppm})$ has been reported. ${ }^{47}$ However, the risk of toxicity caused by $\mathrm{ZnO}$ in our designed system can be excluded since $\mathrm{ZnO}$ NPs have been deposited by spray pyrolysis technique, and a very negligible amount of NPs $(\sim 30 \mu \mathrm{M})$ were found to leach from the glass plate (see Supporting Information, Figure S3) which is a much lower value compared to the in vitro toxic concentration of $\left.\left(\mathrm{Zn}^{2+}\right)_{\mathrm{aq}}(400 \mu \mathrm{M})\right)^{48}$

Since serum albumins are the most abundant proteins in plasma and BR is mostly bound to albumin in blood, we have further extended BR degradation in the presence of HSA protein by using $\mathrm{ZnO}$ NP-based flow-device. The whole system was kept over an UV source, and BR-HSA complex solution was passed through the channel in a very slow flow rate $(2 \mathrm{~mL} /$ $40 \mathrm{~min})$. As shown in Figure 5a, the total amount of BR degradation is found to be $6 \%$ and $51 \%$ in the absence and presence of $\mathrm{ZnO} \mathrm{NPs}$, respectively, under the same flow rate condition. Therefore, it is concluded that BR can be degraded significantly, even if it is bound to HSA protein. Figure $5 \mathrm{~b}$ shows CD spectra of native proteins and the ejected BR-HSA photoproduct, which was obtained after passing HSA-BR complex solution throughout the NP-based flow-device. From the $\mathrm{CD}$ spectra, a very negligible perturbation of the secondary and tertiary (inset) structure of the protein is clearly noticeable.

\section{CONCLUSIONS}

To conclude, we present a novel mechanism for $\mathrm{ZnO}$ NPsensitized BR degradation via the FRET process, as schematically shown in Figure $4 \mathrm{~b}$. The efficient degradation in the presence of smaller $\mathrm{ZnO}$ NPs containing a higher concentration of defect states is attributed to the FRET from NP defect states to surface-attached BR, which introduces a pathway to degrade $\mathrm{BR}$. $\mathrm{ZnO} \mathrm{NP}$ has several advantages for 

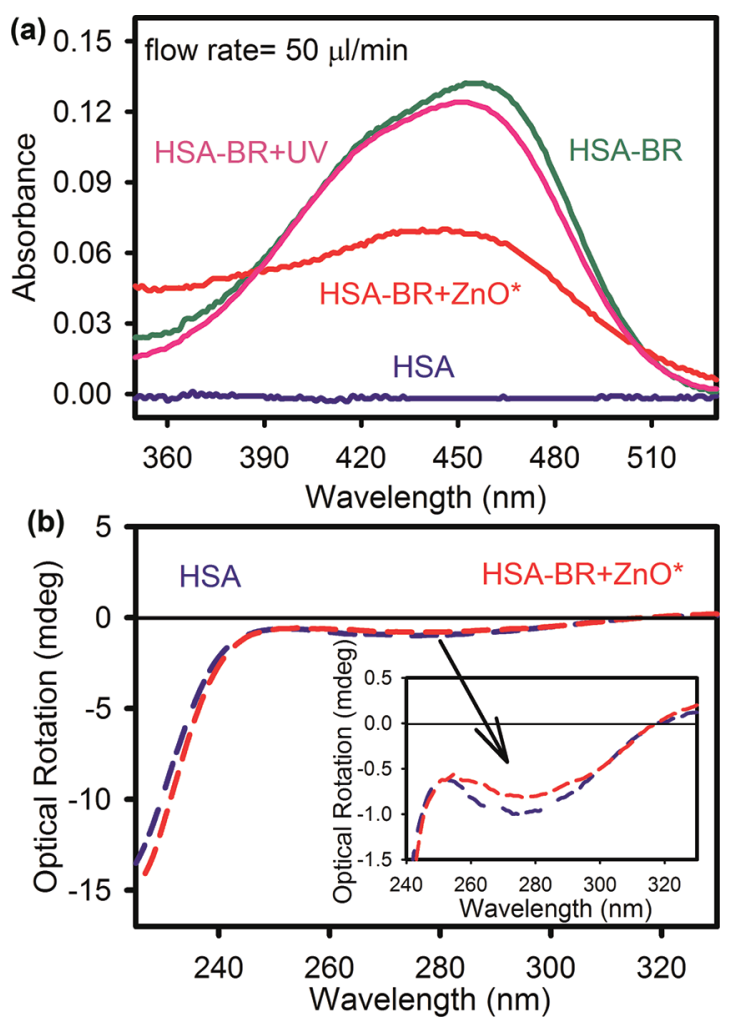

Figure 5. (a) UV-vis spectral changes of BR-HSA complex solution in the absence of UV (green), and upon UV irradiation in the presence (red) and absence (pink) of $\mathrm{ZnO}$ NPs. The absorbance spectrum of HSA is shown for reference (blue). (b) CD spectra of native HSA protein (blue) and HSA-BR complex solution in the presence of UVirradiated $\mathrm{ZnO}$ NPs (red). The near-UV (inset) and far-UV spectra reveal less than $2 \%$ change in tertiary and secondary structures, respectively.

BR photocatalysis under UV irradiation. First, these catalysts are easily prepared, and the method does not need specialized equipment or reagents. Second, these NPs have been demonstrated to work as recyclable photocatalysts and exhibit excellent photocatalytic activity for the degradation of $\mathrm{BR}$ in multiple cycles. Finally, $\mathrm{ZnO}$, which has a huge absorption band in the near-UV range, can protect the harmful effect of UV light. These advantages substantially make this recyclable $\mathrm{ZnO}$ NP photocatalyst promising to be useful in the future technology of BR phototherapy. We have also proposed a FRET-based phototherapeutical device/system involving $\mathrm{ZnO}$ NP thin film in order to degrade BR in blood. Further technological refinement of the $\mathrm{ZnO}$-based "nanotherapy" and rigorous clinical trials are likely to offer better phototherapeutic improvements in the treatment of hyperbilirubinemia.

\section{ASSOCIATED CONTENT}

\section{S Supporting Information}

Investigation of $\mathrm{BR}$ photoproduct and its toxicity, effect of catalyst concentration on the photodegradation of $\mathrm{BR}$, and leaching of $\mathrm{ZnO}$ NPs from flow-device during photocatalysis. This material is available free of charge via the Internet at http://pubs.acs.org.

\section{AUTHOR INFORMATION}

\section{Corresponding Author}

*E-mail address: skpal@bose.res.in.

\section{Notes}

The authors declare no competing financial interest.

\section{ACKNOWLEDGMENTS}

S.S. thanks UGC (India) and A.M. thanks CSIR (India) for fellowships. We thank DST (India) for financial grants SR/SO/ BB-15/2007. S.B., M.A.M., and J.D. would like to acknowledge partial financial support from the National Nanotechnology Center, belonging to the National Science \& Technology Development Agency (NSTDA), Ministry of Science and Technology (MOST), Thailand, and the Centre of Excellence in Nanotechnology at the Asian Institute of Technology, Thailand. We thank the learned reviewers for their constructive comments to improve the quality of the work.

\section{REFERENCES}

(1) Ostrow, J. D. Bile Pigments and Jaundice: Molecular, Metabolic and Medical Aspects; Marcel Dekker: New York, 1986.

(2) Stocker, R.; Glazer, A. N.; Ames, B. N. Proc. Natl. Acad. Sci. U.S.A. 1987, 84, 5918-5922.

(3) Lightner, D. A.; McDonagh, A. F. Acc. Chem. Res. 1984, 17, 417424.

(4) Lamola, A. A.; Blumberg, W. E.; McClead, R.; Fanaroff, A. Proc. Natl. Acad. Sci. U.S.A. 1981, 78, 1882-1886.

(5) Braslavsky, S. E.; Holzwarth, A. R.; Schaffner, K. Angew. Chem., Int. Ed. 1983, 22, 656-674.

(6) Agati, G.; Fusi, F.; Pratesi, R.; McDonagh, A. F. Photochem. Photobiol. 1992, 55, 185-190.

(7) McDonagh, A. F.; Agati, G.; Fusi, F.; Pratesi, R. Photochem. Photobiol. 1989, 50, 305-319.

(8) McDonagh, A. F.; Palma, L. A.; Lightner, D. A. Science 1980, 208, 145-151.

(9) Zhang, J. Z. J. Phys. Chem. Lett. 2010, 1, 686-695.

(10) Paciotti, G. F.; Myer, L.; Weinreich, D.; Goia, D.; Pavel, N.; McLaughlin, R. E.; Tamarkin, L. Drug Delivery 2004, 11, 169-183.

(11) Wu, X.; Liu, H.; Liu, J.; Haley, K. N.; Treadway, J. A.; Larson, J. P.; Ge, N.; Peale, F.; Bruchez, M. P. Nat. Biotechnol. 2002, 21, 41-46.

(12) Hirsch, L. R.; Stafford, R. J.; Bankson, J. A.; Sershen, S. R.; Rivera, B.; Rrice, R. E.; Hazle, J. D.; Halas, N. J.; West, J. L. Proc. Natl. Acad. Sci. U.S.A. 2003, 100, 13549-13554.

(13) Topoglidis, E.; Campbell, C. J.; Cass, A. E. G.; Durrant, J. R. Langmuir 2001, 17, 7899-7906.

(14) Li, Y.; Xie, W.; Hu, X.; Shen, G.; Zhou, X.; Xiang, Y.; Zhao, X.; Fang, P. Langmuir 2010, 26, 591-597.

(15) Zhou, J.; Xu, N. S.; Wang, Z. L. Adv. Mater. 2006, 18, 24322435.

(16) Kamat, P. V.; Huehn, R.; Nicolaescu, R. J. Phys. Chem. B 2002, $106,788-794$.

(17) Herrmann, J. M. Catal. Today 1999, 53, 115-129.

(18) Maness, P. C.; Smolinski, S.; Blake, D. M.; Huang, Z.; Wolfrum, E. J.; Jacoby, W. A. Appl. Environ. Microbiol. 1999, 65, 4094-4098.

(19) Sugunan, A.; Warad, H. C.; Boman, M.; Dutta, J. J. Sol-Gel Sci. Technol. 2006, 39, 49-56.

(20) Lakowicz, J. R. Principles of Fluorescence Spectroscopy, 2nd ed.; Kluwer Academic/ Plenum: New York, 1999.

(21) Braslavsky, S. E.; Fron, E.; Rodriguez, H. B.; Roman, E. S.; Scholes, G. D.; Schweitzer, G.; Valeur, B.; Wirz, J. Photochem. Photobiol. Sci. 2008, 7, 1444-1448.

(22) Sinha, S. S.; Verma, P. K.; Makhal, A.; Pal, S. K. Rev. Sci. Instrum. 2009, 80, 053109.

(23) Wang, X.; Kong, X.; Yu, Y.; Zhang, H. J. Phys. Chem. C 2007, $111,3836-3841$.

(24) Ye, J. D.; Gu, S. L.; Qin, F.; Zhu, S. M.; Liu, S. M.; Zhou, X.; Liu, W.; Hu, L. Q.; Zhang, R.; Shi, Y.; Zheng, Y. D. Appl. Phys. A: Mater. Sci. Process. 2005, 81, 759-762.

(25) Idriss, H.; Barteau, M. A. J. Phys. Chem. 1992, 96, 3382-3388. 
(26) Idriss, H.; Andrews, R. M.; Barteau, M. A. J. Vac. Sci. Technol. A

1993, 11, 209-218.

(27) Clegg, R. M. Methods Enzymol. 1992, 211, 353-388.

(28) Lilley, D. M. J.; Wilson, T. J. Curr. Opin. Chem. Biol. 2000, 4, 507-517.

(29) Warner, J. H.; Watt, A. R.; Thomsen, E.; Heckenberg, N.; Meredith, P.; Rubinsztein-Dunlop, H. J. Phys. Chem. B 2005, 109, 9001-9005.

(30) Makhal, A.; Sarkar, S.; Bora, T.; Baruah, S.; Dutta, J.; Raychaudhuri, A. K.; Pal, S. K. Nanotechnology 2010, 21, 265703.

(31) Baruah, S.; Sinha, S. S.; Ghosh, B.; Pal, S. K.; Raychaudhuri, A. K.; Dutta, J. J. Appl. Phys. 2009, 105, 074308.

(32) Sarkar, S.; Makhal, A.; Bora, T.; Baruah, S.; Dutta, J.; Pal, S. K. Phys. Chem. Chem. Phys. 2011, 13, 12488-12496.

(33) Yang, Z.; Zhang, C. J. Mol. Catal. A: Chem. 2009, 302, 107-111.

(34) Kurtin, W. E. Photochem. Photobiol. 1978, 27, 503-509.

(35) Narayanan, R.; El-Sayed, M. A. Nano Lett. 2004, 4, 1343-1348.

(36) Mclaren, A.; Solis, T. V.; Li, G.; Tsang, S. C. J. Am. Chem. Soc. 2009, 131, 12540-12541.

(37) Auffan, M.; Rose, J.; Bottero, J. Y.; Lowry, G. V.; Jolivet, J. P.; Wiesner, M. R. Nat. Nanotechnol. 2009, 4, 634-641.

(38) Burda, C.; Chen, X. B.; Narayanan, R.; El-Sayed, M. A. Chem. Rev. 2005, 105, 1025-1102.

(39) Murdoch, M.; Waterhouse, G. I. N.; Nadeem, M. A.; Metson, J. B.; Keane, M. A.; Howe, R. F.; Llorca, J.; Idriss, H. Nat. Chem. 2011, 3, 489-492.

(40) Wang, J. C.; Liu, P.; Fu, X. Z.; Li, Z. H.; Han, W.; Wang, X. X. Langmuir 2009, 25, 1218-1223.

(41) Guo, M. Y.; Ng, A. M. C.; Liu, F.; Djuri, A. B.; Chan, W. K.; Su, H.; Wong, K. S. J. Phys. Chem. C 2011, 115, 11095-11101.

(42) Bohle, D. S.; Spina, C. J. J. Am. Chem. Soc. 2009, 131, 43974404.

(43) Hoffmann, M. R.; Martin, S. T.; Choi, W. Y.; Bahnemann, D. W. Chem. Rev. 1995, 95, 69-96.

(44) Xu, Y.; Langford, C. H. J. Photochem. Photobiol. A 2000, 133, $67-71$.

(45) Das, J.; Khushalani, D. J. Phys. Chem. C 2010, 114, 2544-2550.

(46) Kuo, T. J.; Lin, C. N.; Kuo, C. L.; Huang, M. H. Chem. Mater. 2007, 19, 5143-5147.

(47) Brunner, T. J.; Wick, P.; Manser, P.; Spohn, P.; Grass, R. N.; Limbach, L. K.; Bruinink, A.; Stark, W. J. Environ. Sci. Technol. 2006, 40, 4374-4381.

(48) Palmiter, R. D. Proc. Natl. Acad. Sci. U.S.A. 2004, 101, 49184923. 